\title{
Analysis of Enterprise Strategic Management Issues and Coping Strategies Based on Big Data Analysis
}

\author{
Ziqi ZHONG ${ }^{1, \mathrm{a}^{*}}$, Wang Haoran ${ }^{2}$, WANG JUNSHENG ${ }^{3}$ \\ ${ }^{1}$ Department of International Business, Graduate School of Pan-Pacific International Studies, Kyung Hee University, Yongin-Si, \\ Gyeonggi-Do, South Korea \\ ${ }^{2}$ Department of International Business, Graduate School of Pan-Pacific International Studies, Kyung Hee University, Yongin-Si, \\ Gyeonggi-Do, South Korea \\ ${ }^{3}$ Department of International Business, Graduate School of Pan-Pacific International Studies, Kyung Hee University, Yongin-Si, \\ Gyeonggi-Do, South Korea
}

\begin{abstract}
Corporate strategic management is an important management mode that affects the development of an enterprise. It plays a very important role in the development of corporate strategic management. In recent years, information technology has developed rapidly, data is frequently updated, and huge amounts of data are generated every day. Social development has entered the era of big data, which makes enterprises face more opportunities and challenges in formulating strategies and operating management. In order to enable enterprises to adapt to the development of the times and obtain healthy and sound development results. This article analyzes and summarizes the new characteristics of enterprise management in the context of big data, and applies big data analysis technology to the environmental analysis of enterprises, and points out the problems of strategic management of enterprises in the context of big data. This article aims at the current problems and proposes specific strategies after in-depth research, which provides a reference basis for strategic management of enterprises in the era of big data. It has certain practical significance and can help Chinese enterprises quickly adapt to the new environment.
\end{abstract}

\section{Introduction}

Big data, as the Internet, the Internet of Things, mobile computing, and cloud computing, is another disruptive technological change in the IT industry. It is redefining the processes and methods of social management and national strategic decisions, corporate management decisions, and personal decisions [1]. McKinsey \& Company said in a research report that according to Western industry data, the application of big data will save governments in developed European countries more than 100 billion euros in operating costs, and reduce the cost of the U.S. healthcare industry by $8 \%$, approximately every year More than 300 billion U.S. dollars, and increased retailers' operating margins by more than $60 \%$. According to the research report of the "Digital Universe" of market research organization IDC, the big data technology and service market will reach $\$ 16.9$ billion in 2015 , achieving an annual growth rate of $40 \%$, which is 7 times the growth rate of the IT and communications industry [2]. Big data contains great business value, scientific research value, etc. This value of big data is currently being recognized and exploited. Therefore, research on management and decisionmaking driven by big data not only has outstanding scientific frontiers and great strategic significance, but also has great practical value and distinctive characteristics of the times [3].

This article starts with the new features and new challenges faced by the Institute of Management and Decision in the Big Data environment, and analyzes how Big Data technology can serve strategic management of enterprises, with a view to providing support for academic innovation in the field of management and decision-making in the era of Big Data. Reference, and provide assistance for China's leap forward in the development wave of big data technologies and applications [4].

\section{New features of corporate strategic management in the context of big data}

The "big data" technique first appeared in 2008, and it aroused widespread research interest in the academic community. "Nature" and "Science" magazines respectively publish special issues to discuss big data processing and application topics from multiple aspects, including Internet technology, supercomputing, Internet economics and other aspects. Governments of all countries attach great importance to research and

*Corresponding author's e-mail: achichung@khu.ac.kr 
exploration in the field of big data, and carry out research planning at the national strategic level. In the research and application of big data, it has gradually begun to face management and decision-making, and research concepts and methods have been fully developed [5].

In the context of big data, government decisionmaking, industrial policy, and macro-management activities are mostly characterized by high-frequency real-time, deep customization, and multi-agent decision making. This requires the design and construction of corresponding management decision analysis models and methods that effectively combine information science and business applications [6]. Under the market mechanism of data generation, collaborative innovation work is carried out. In the context of big data, new research and applications related to management and decision-making present new characteristics: at the economic and social level, the rapid development of big data has enabled the operation of economic and social systems, government evaluations, and so on. Flow and utilization are more dependent; At the industry level, many industries have been impacted by the integration of big data, and new models have been spawned in areas such as business and finance; At the individual level such as people and organizations, the development of big data has promoted comprehensive online, offline integration, extensive connections and high-frequency interaction; At the level of information technology, big data for management decision-making presents a series of characteristics such as wide distribution, huge scale, and diverse modalities; At the level of computing theory, big data for management decision-making presents characteristics such as non-random acquisition, irregularity, and alienation structure [7].

\section{Enterprise strategic management process}

The strategic management process is the overall deployment of a company's strategic management issues. The strategic management process consists of three parts. Among them, strategic analysis includes analysis of the internal and external environment of the enterprise; Strategic choices are divided into company strategy, competition strategy, internationalization strategy, etc. This article is based on the theory of corporate strategic management process as the overall analysis framework to carry out research. Figure 1 shows the relationship between the elements in the strategic management process [8].

\subsection{Business strategy analysis}

Strategic analysis is the first stage of the strategic management process. Enterprise strategic management is generally divided into analysis of the external environment of the enterprise and analysis of the internal conditions of the enterprise.

\subsection{Corporate Strategy Choice}

Strategic selection can be called a strategic decisionmaking process for enterprises, which is a dynamic process. There are many ways to choose a company's strategy, including company strategy, competitive strategy, and international strategy.

\subsection{Corporate Strategy Implementation}

The implementation of corporate strategy refers to the analysis of the internal and external environment of the enterprise. Based on the strategic choices, the enterprise implements the formulated strategic plan and achieves the expected strategic goals. During the implementation of corporate strategy, enterprises need to give play to their own advantages, make use of their own resources, and finally reach their expected goals.

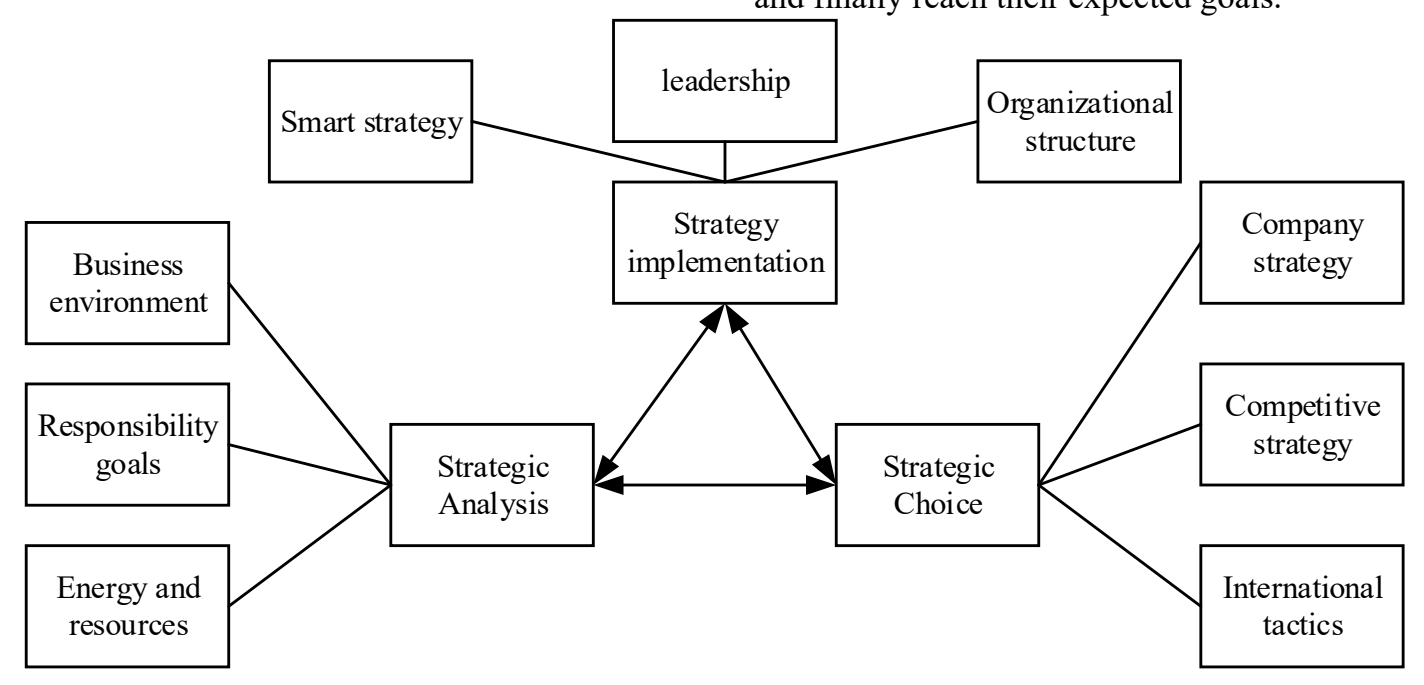

Figure 1. Strategic management process 


\section{Application of big data analysis technology to the analysis of corporate competition environment for strategic management}

\subsection{Contents of Enterprise Competitive Environment Analysis}

Big data provides rich possibilities for modeling, forecasting, and optimizing management systems. In a narrow sense, analysis of the competitive environment of an enterprise generally includes analysis of competitors and analysis of the competitive landscape (strategic groups within the industry). This section uses big data analysis to analyze the competitive environment.

Competitor analysis mainly has four aspects, which are the competitors' future goals, assumptions, current strategies and potential capabilities. The content of competitor analysis will provide a basis for data collection for big data analysis.

Another aspect of the analysis of the competitive environment is to determine the characteristics of all major competitors in the industry and classify them based on this characteristic, that is, strategic group analysis. Products and services, market segmentation, integration level, marketing intensity, price level, cost positioning, organization size, etc. can all be used as characteristics to distinguish strategic groups. Strategic group analysis helps companies understand their own strategic position relative to other companies and the competitive effects that a company's strategic changes may cause. The content and methods of competitor analysis will provide the basis for the selection of information sources, the determination of topics, the selection of methods and the analysis of results in the data mining process.

\subsection{Process of enterprise competition environment analysis based on big data analysis technology}

The brief process of applying big data technology to the analysis of the competitive environment of an enterprise is shown in Figure 2, which includes defining problems based on the content of the competitive environment analysis, identifying information sources, collecting and organizing data, data storage systems, data mining, and analyzing and expressing results.

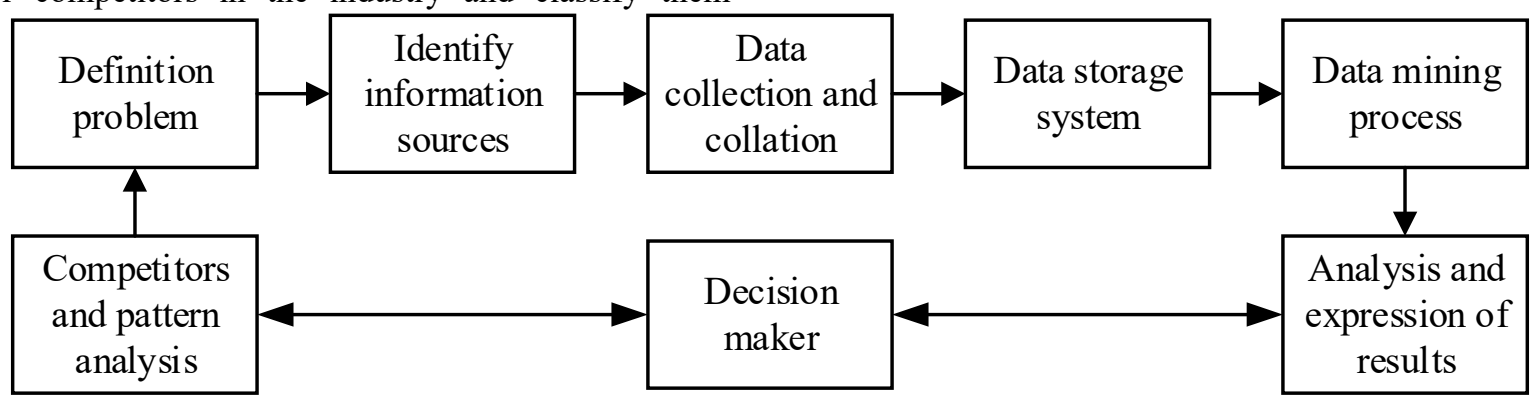

Figure 2. Brief process of enterprise competition environment analysis based on big data analysis technology

Most companies already have a lot of information about competitors, for example, information resources commonly used in production and management of enterprises include: e-mail, spreadsheets, text files, flat files, XML content and voice mail, various types of contracts, product specifications , Survey reports, information from business partners, website information, and other structured, semi-structured, and unstructured information resources. There is a lot of data, it is relatively scattered, and most of it is useless data. How to extract effective data from these massive and disorganized data and discover its potential value is the most basic function of big data technology. According to the survey, $80 \%$ of the competitive intelligence required by an enterprise can be obtained from within the enterprise, so internal information is an information source that requires special attention in the analysis of the competitive environment. In general, the key to digging and utilizing internal information resources of an enterprise is to be familiar with the characteristics of the work of various departments within the enterprise, to understand the information that each department may have, and to organize and process it.
In the age of information explosion, the information available to corporate managers has never been so rich, complex, unstructured and difficult to understand. This includes economic, social and environmental information from internal and external channels, financial and non-financial. In the analysis of the strategic competitive environment of enterprises, the use of big data technology can effectively analyze the competitors and the industrial structure. Technical methods such as clustering, association rules, web mining, and text mining in big data analysis can be effectively applied to the analysis of corporate competitive environment. A brief example is shown in Table 1. 
TABLE I. EXAMPLES OF DATA MINING METHODS APPLIED TO COMPETITIVE ENVIRONMENT ANALYSIS

\begin{tabular}{|c|c|}
\hline $\begin{array}{l}\text { Big data analysis } \\
\text { methods }\end{array}$ & $\begin{array}{l}\text { Application examples in the } \\
\text { analysis of corporate } \\
\text { competitive environment }\end{array}$ \\
\hline $\begin{array}{l}\text { Clustering (K-means, } \\
\text { Kohonen, etc.) }\end{array}$ & $\begin{array}{l}\text { Effectively cluster the } \\
\text { competitive environment } \\
\text { information, such as classifying } \\
\text { competitors into different } \\
\text { categories through clustering } \\
\text { when the eigenvalues are } \\
\text { unknown; automatically } \\
\text { identifying strategic groups in } \\
\text { the industry; using consumer } \\
\text { data to analyze customer loyalty } \\
\text { through clustering, etc. }\end{array}$ \\
\hline $\begin{array}{l}\text { Association rule } \\
\text { analysis (Apriori, } \\
\text { GRI, etc.) }\end{array}$ & $\begin{array}{l}\text { Discover relevant rules in } \\
\text { competitive environment } \\
\text { information, such as } \\
\text { discovering correlations } \\
\text { between competitors' different } \\
\text { products }\end{array}$ \\
\hline $\begin{array}{l}\text { Classification (K } \\
\text { nearest neighbor } \\
\text { classification, C5.0 } \\
\text { decision tree, artificial } \\
\text { neural network, } \\
\text { support vector } \\
\text { machine, Bayesian } \\
\text { classification, etc.) }\end{array}$ & $\begin{array}{l}\text { Such as classifying competitors } \\
\text { based on a certain characteristic } \\
\text { value (market behavior, type of } \\
\text { competitive strategy, etc.); } \\
\text { decision trees can provide } \\
\text { decision references in the form } \\
\text { of a tree structure or "if, then" }\end{array}$ \\
\hline Web mining & $\begin{array}{l}\text { Information about competitors } \\
\text { can be extracted from the } \\
\text { Internet; }\end{array}$ \\
\hline Text mining & $\begin{array}{l}\text { Use text classification } \\
\text { technology to quickly and } \\
\text { efficiently categorize a large } \\
\text { number of documents about the } \\
\text { competitive environment; find } \\
\text { the connection between related } \\
\text { content in different information } \\
\text { sources }\end{array}$ \\
\hline $\begin{array}{l}\text { Fuzzy data mining } \\
\text { method }\end{array}$ & $\begin{array}{l}\text { Evaluate competitive } \\
\text { environment information; } \\
\text { identify potential competitors; } \\
\text { predict competitors' behavior, } \\
\text { etc. }\end{array}$ \\
\hline
\end{tabular}

The big data analysis technology sorts out the information related to the analysis of the competitive environment of the enterprise in the huge database, and obtains useful data. The results can be visually presented to users through data visualization techniques. Managers at different levels of the company make relevant decisions based on this knowledge, investigate and analyze the strength of competitors and the competitive landscape that the company faces, and provide support for the company's strategy formulation and implementation.

\section{Problems in corporate strategic management in the context of big data}

\subsection{Enterprise managers don't pay enough attention to big data}

The popularization of big data technology has been rapid, which has played a good role in promoting the development of enterprises. However, corporate management still has cognitive limitations on big data, which severely restricts the management efficiency and the overall development of the industry. Connect with the latest Internet thinking. In practice, especially the leaders of state-owned enterprises and private enterprises are not able to effectively use data resources in decisionmaking. They mainly make head decisions and do not use the effective information brought by data effectively.

\subsection{Enterprises lack big data talent}

Big data technology has high requirements for employees' computer level and statistical knowledge. Under the current resource allocation conditions, most of the small and medium-sized traditional enterprises in China still do not have the ability to perform big data decision analysis. On the one hand, the existing server and equipment resources of the enterprise cannot store and continuously obtain high-value data, and it is difficult to obtain first-hand information; On the other hand, due to the high requirements of big data on employees, traditional enterprises also generally face the problem of lack of data talents, unable to extract valuable information from redundant data, lack of big data management skills, and important management decisions still need Managers make subjective judgments and decisions.

\subsection{Big data technology is still in the research stage}

Although the big data technology in Europe and the United States is relatively mature, it has accumulated many advanced concepts and knowledge, and has been applied in many large enterprises and achieved good results. After the introduction of foreign advanced big data theory in China, many high-end talents and professionals have expressed strong support for this, and based on this, they have carried out many more in-depth studies that are in line with China's social characteristics and promote corporate management innovation. However, due to human and financial constraints, many traditional small companies are not able to apply the research results of advanced big data technology to daily business management. 


\section{Analysis of enterprise strategic management countermeasures based on big data analysis}

\subsection{Changes in strategic management thinking}

Actively incorporating big data thinking into the corporate strategic management decision-making process is a necessary way to change the traditional business management model. First of all, enterprises should adjust the management's cognitive approach, introduce more new management talents, bring new management ideas and experiences, and innovate existing management models in combination with big data technology. Secondly, it provides regular information and industry training for enterprise employees, and strives to help leaders' ideological level and professional ability to keep pace with the development of the big data era, so as to provide effective suggestions for corporate strategy formulation and decision-making issues. Finally, companies can use brainstorming and full participation discussions to motivate all employees to participate in discussions on future strategic development directions and related development issues, adjust existing management ideas, and stimulate creativity and vitality.

\subsection{Introducing big data talent}

At this stage, companies should increase their focus on big data management talents and technical talents, and attract and retain more big data talents through reasonable recruitment and training promotion programs. Big data talents are one of the important influencing factors of strategic management upgrade of enterprises. In the context of economic development in the new era, enterprises should set up a professional big data management department. The staff team must have good data architecture analysis capabilities, and use various algorithms and technical methods to mine more valuable information from massive amounts of information. Assist sales and research and development of corporate products, and keep pace with corporate strategic management. Unlike the data in the traditional era, the amount of data information in the big data era is relatively large, requiring companies to purchase professional servers and computer equipment, and only professional personnel can do the job well. Therefore, enterprises need to speed up the introduction of talents, inject new ways of thinking into existing work teams, and at the same time, they should increase the trust of existing employees in the company; In addition, companies also need to conduct regular training for existing technical department employees to encourage employees to mine corporate information and provide guidance to management.

\subsection{Improve big data utilization}

Enterprises can extract valuable key information from a large amount of complex structured data, and conduct effective in-depth analysis of this in combination with market business and operating activities to extract the development law of the market industry and commodities. It can be seen that enterprises should improve the utilization efficiency of big data resources. For example, for companies that include sales services, in order to improve the rationality of the sales management strategy. On the one hand, it is necessary to obtain and actively collect user purchase behavior data, understand the user's purchase preferences, and provide personalized recommendation services; On the other hand, business analysis staff should analyze the potential demand of the market and predict the future development trend of the product, so as to better formulate the production and sales plan.

\section{Conclusion}

This article from the perspective of corporate strategic management, combined with current big data technology, applies big data analysis technology to corporate strategic management, improves the efficiency of corporate strategic management, and brings new opportunities to corporate strategic management; At the same time, some new problems have emerged. Aiming at the existing problems, this paper proposes strategies to solve these problems, which has a certain reference role for the construction of the management team and has practical significance. At this stage, enterprises should actively use big data technology to assist their strategic decisions, improve their strategic management efficiency, increase their market competitiveness, and promote their healthy development.

\section{References}

1. W. K. Bao, An Analysis of the Evolution of Strategic Management Thoughts in the Andrews Framework, Qilu Petrochemical, 2005, pp.236-238.

2. B. Dai, The Harmonious Connotation of Contemporary Transcending Competition Strategy, Journal of Shijiazhuang University of Economics, 2010, vol. 33, pp.77-79.

3. H. Chen, Strategy determines the future-On the strategy of small and medium-sized enterprises, Science \& Technology, 2011, vol. 9X, pp.6-6.

4. X. H. Yang, J. Wang, Strategic Management of Enterprises: Theory and Cases, Higher Education Press, 2016.

5. J. H. Zhou, Research on the matching relationship between corporate culture and corporate strategic management, Economist, 2015, vol.4, pp. 276-277.

6. X. Tao, Understanding the Construction of Competitive Advantage in the Implementation of 
Enterprise Strategy, Exploration of Economic Issues, 2013, vol.3, pp.158-161.

7. C. H. Zhang, Practical Thoughts on Problems, Analysis and Countermeasures of Enterprise Strategic Management, National Commercial
Conditions - Theoretical Research, 2014, vol.1, pp.44-46.

8. X. X. Yao, F. Zhang, L. Sun, Application of Big Data Technology in Enterprise Strategic Management, Electronic Technology and Software Engineering, 2017, vol.14, pp.170- 170. 